\title{
Public Census Data on CD-ROM at Lawrence Berkeley Laboratory
}

\author{
by \\ LBL-32165-ReV. 1 \\ DE93 001589 \\ Deane W. Merrill
}

Information and Computing Sciences Division

Lawrence Berkeley Laboratory

University of California

Berkeley, California 94720

April 1992

revised 2 July 1992

This work was supported by the Director, Office of Epidemiology and Health Surveillance, Office of Health, Office of Environment, Safety and Health, U.S. Department of Energy under Contract No. DE-AC0376SF00098.

\section{MASTER}




\section{INTRODUCTION}

The Comprehensive Epidemiologic Data Resource (CEDR) and Populations at Risk to Environmental Pollution (PAREP) projects, of the Information and Computing Sciences Division (ICSD) at Lawrence Berkeley Laboratory (LBL), are using public socio-economic and geographic data files which are available to CEDR and PAREP collaborators via LBL's computing network. At this time 67 CD-ROM diskettes (approximately 35 gigabytes) are on line via the Unix file server cedrcd.lbl.gov. Most of the files are from the U.S. Bureau of the Census, and most pertain to the 1990 Census of Population and Housing.

All the CD-ROM diskettes contain documentation in the form of ASCII text files. In addition, printed documentation for most files is available for inspection at University of California Data and Technical Assistance (UC DATA), tel. (510) 642-6571, or the UC Documents Library, tel. (510) 642-2569, botk: located on the UC Berkeley Campus. Many of the CD-ROM diskettes distributed by the Census Bureau contain software for PC compatible computers, for easily accessing the data.

Shared access to the data is maintained through a collaboration among the CEDR and PAREP projects at LBL, and UC DATA, and the UC Docisments Library. LBL is grateful to UC DATA and the UC Documents Library for the use of their CD-ROM diskettes. Continued shared access in the future is not guaranteed if costs become prohibitive.

Via the Sun Network File System (NFS), these data can be exported to Internet computers for direct access by the user's application program(s). Due to the size of the files, this access method is preferred over File Transfer Protocol (FTP) access. Please contact Deane Merrill ( dwmerrillabl.gov) if you wish to make use of the data. 


\section{SUMMARY OF DATA ON CD-ROM}

The table below summarizes data on CD-ROM currently available on line (location = path name in cedrcd.1bl.gov; disks = number of CD-ROM diskettes; $M B=$ size in megabytes.) Most of the CD-ROM diskettes have their own directory structure, including separate files for documentation and different types of data.

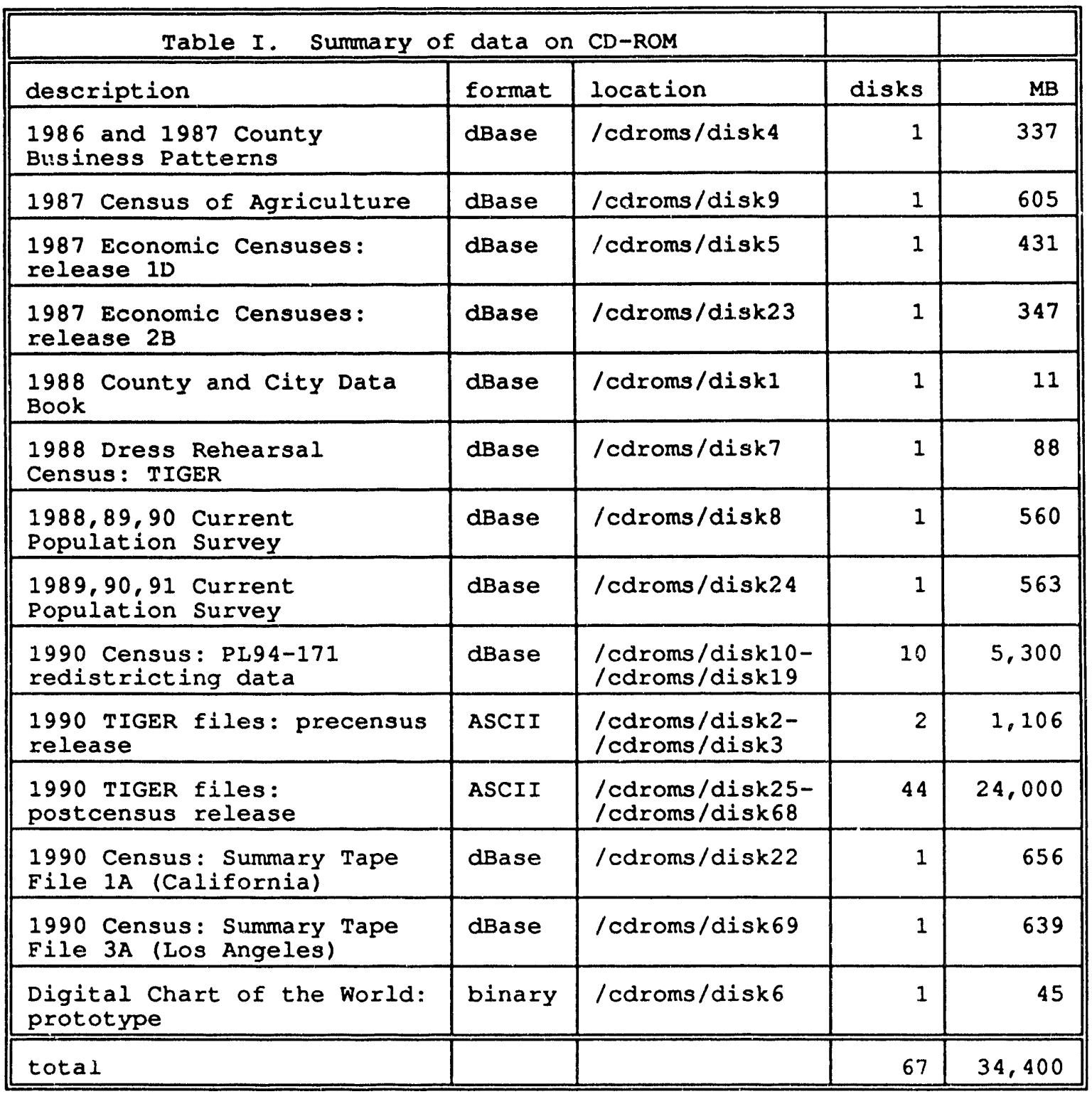




\section{CENSUS PL94-171 REDISTRICTING DATA}

The locations of 1990 Census PL94-171 redistricting data, by state, are as follows. (FIPS = Federal Information Processing System state code; location = path name in cedrcd. lbl.gov; $\mathrm{MB}=$ size in megabytes; ncy = number of counties.)

\begin{tabular}{|c|c|c|c|c|c|}
\hline state & $a b b r$ & FIPS & location & $\mathrm{MB}$ & ncy \\
\hline United States & & & & 5,000 & 3,141 \\
\hline Alabama & AL & 01 & /cdroms/disk13/p19417al.* & 119 & 67 \\
\hline Alaska & AK & 02 & /cdroms/disk12/p19417ak.* & 14 & 25 \\
\hline Arizona & $\mathrm{Az}$ & 04 & /cdroms/disk10/p19417az. * & 75 & 15 \\
\hline Arkansas & AK & 05 & /cdroms/disk14/p19417ar.* & 98 & 75 \\
\hline California & $\mathrm{CA}$ & 06 & /cdroms/disk11/pl9417ca.* & 357 & 58 \\
\hline Colorado & $\mathrm{Co}$ & 08 & /cdroms/disk12/p19417co.* & 88 & 63 \\
\hline Connecticut & $\mathrm{CT}$ & 09 & /cdroms/disk15/pl9417ct.* & 38 & 8 \\
\hline Delaware & $\mathrm{DE}$ & 10 & /cdroms/disk13/pl9417de.* & 11 & 3 \\
\hline $\begin{array}{l}\text { District of } \\
\text { Columbia }\end{array}$ & DC & 11 & /cdroms/disk15/p19417dc.* & 4 & 1 \\
\hline Florida & FL & 12 & /cdroms/disk16/p19417fl.* & 220 & 67 \\
\hline Georgia & GA & 13 & /cdroms/disk10/p19417ga.* & 142 & 159 \\
\hline Hawaii & HI & 15 & /cdroms/disk17/p19417hi.* & 11 & 5 \\
\hline Idaho & ID & 16 & /cdroms/disk12/p19427id.* & 41 & 44 \\
\hline Illinais & IL & 17 & /cdroms/disk13/p19417il.* & 246 & 102 \\
\hline Indiana & IN & 18 & /cdroms/disk14/p19417in.* & 147 & 92 \\
\hline Iowa & $1 \mathrm{~A}$ & 19 & /cdroms/disk18/p19417ia.* & 111 & 99 \\
\hline Kansas & ks & 20 & /cdroms/disk18/p19417ks.* & 118 & 105 \\
\hline Kentucky & KY & 21 & /cdroms/disk16/p19417ky.* & 84 & 120 \\
\hline Louisiana & LA & 22 & /cdroms/disk14/p194171a.* & 109 & 64 \\
\hline Maine & ME & 23 & /cdroms/disk12/p19417me.* & 36 & 16 \\
\hline Maryland & $\mathrm{MD}$ & 24 & /cdroms/disk15/p19417md.* & 59 & 24 \\
\hline Massachusetts & MA & 25 & /cdroms/disk16/p19417ma.* & 79 & 14 \\
\hline Michigan & MI & 26 & /cdroms/disk10/p19417mi.* & 181 & 83 \\
\hline Minnesota & MN & 27 & /cdroms/disk18/p19417mn.* & 130 & 87 \\
\hline Mississippi & MS & 28 & /cdroms/disk14/p19417ms.* & 79 & 82 \\
\hline
\end{tabular}




\begin{tabular}{|c|c|c|c|c|c|}
\hline state & $a b b r$ & FIPS & location & MB & ncy \\
\hline Missouri & Mo & 29 & /cdroms/disk17/pl9417mo.* & 152 & 115 \\
\hline Montana & $\mathrm{MT}$ & 30 & /cdroms/disk17/p19417mt.* & 40 & 57 \\
\hline Nebraska & NE & 31 & /cdroms/disk13/pl9417ne.* & 81 & 93 \\
\hline Nevada & $\mathrm{NV}$ & 32 & /cdroms/disk17/p19417nv.* & 25 & 17 \\
\hline New Hampshire & NH & 33 & /cdroms/disk10/p19417nh.* & 23 & 10 \\
\hline New Jersey & NJ & 34 & /cdroms/disk19/pl9417nj.* & 106 & 21 \\
\hline New Mexico & NM & 35 & /cdroms/disk16/pl9417nm.* & 56 & 33 \\
\hline New York & NY & 36 & /cdroms/disk11/p19417ny.* & 231 & 62 \\
\hline North Carolina & NC & 37 & /cdroms/disk15/pl9417nc.* & 158 & 100 \\
\hline North Dakota & ND & 38 & /cdroms/disk10/pl9417nd. * & 62 & 53 \\
\hline Ohio & $\mathrm{OH}$ & 39 & /cdroms/disk15/p19417oh.* & 180 & 88 \\
\hline Oklahoma & OK & 40 & /cdroms/disk13/pl9417ok. * & 116 & 77 \\
\hline Oregon & OR & 41 & /cdroms/disk13/p19417or.* & 69 & 36 \\
\hline Pennsylvania & PA & 42 & /cdroms/disk18/p19417pa.* & 241 & 67 \\
\hline Rhode Island & RI & 44 & /cdroms/disk15/p19417ri.* & 15 & 5 \\
\hline South Carolina & sc & 45 & /cdroms/disk12/pl9417sc.* & 99 & 46 \\
\hline South Dakota & SD & 46 & /cdroms/disk14/p19417sd.* & 52 & 66 \\
\hline Tennessee & $\mathrm{TN}$ & 47 & /cdroms/disk16/p19417tn.* & 121 & 95 \\
\hline Texas & $T X$ & 48 & /cdroms/disk17/p19417tx, * & 345 & 254 \\
\hline Utah & UT & 49 & /cdroms/disk16/pl9417ut.* & 37 & 29 \\
\hline Vermont & VT & 50 & /cdroms/disk19/pl9417vt.* & 16 & 14 \\
\hline Virginia & VA & 51 & /cdroms/disk19/pl9417va.* & 106 & 136 \\
\hline Washington & WA & 53 & /cdroms/disk12/p19417wa.* & 96 & 39 \\
\hline West Virginia & wV & 54 & /cdroms/disk12/pl9417wv.* & 56 & 55 \\
\hline Wisconsin & WI & 55 & /cdroms/disk10/p19417wi.* & 139 & 72 \\
\hline Wyoming & WY & 56 & /cdroms/disk14/p19417wy.* & 37 & 23 \\
\hline
\end{tabular}




\section{TIGER FILES: PRECENSUS RELEASE}

The precensus TIGER files have been replaced by postcensus files (see Table IV). As of 6/29/92, precensus TIGER files for California and Hawaii are temporarily on line in the locations indicated. (FIPS = Federal Information Processing System state code;

location = path name in cedrcd. l.bl.gov ; $M B=$ size in megabytes; ncy = number of counties.)

Geographic reference name files are located in /cdroms/disk2/grf_n_06 and /cdroms/disk3/grf_n_06 . File sizes listed under "MB" do not include geographic reference name files.

\begin{tabular}{||c|c|c|c|r|r||}
\hline \multicolumn{5}{|c|}{ Table III. 1990 TIGER files: precensus release } \\
\hline \hline $\begin{array}{c}\text { state / } \\
\text { counties }\end{array}$ & abbr & FIPS & location & MB & ncy \\
\hline \hline \begin{tabular}{c} 
California \\
\hdashline-1 Alameda-Plumas \\
Riverside-Yuba
\end{tabular} & CA & 06 & total & 1,106 & 58 \\
\hline
\end{tabular}

\section{TIGER FILES: POSTCENSUS RELEASE}

The locations of postcensus TIGER files, by state, are as follows. (FIPS = Federal Information Processing System state code; call = call number in UC Berkeley Documents Library catalog; location = path name in cedrcd. $1 \mathrm{bl} . \mathrm{gov} ; \mathrm{MB}=$ size in megabytes; ncy $=$ number of counties.) Full call number $=$ H A 2011990 T53 COMPU/D <Call> DOCS where $\langle$ call 1$\rangle=1$ through 44 .

Geographic reference name files are located in /cdroms/disk26/pubgrf90.s01, etc. Files listed under "MB" do not include geographic reference name files.

\begin{tabular}{||l|l|l|l|l|r|r||}
\hline \multicolumn{7}{|c|}{ Table IV. 1990 TIGER files: postcensus release } \\
\hline \hline state / counties & abbr & FIPS & call & location & MB & ncy \\
\hline \hline United States & & & & total & 24,000 & 3,141 \\
\hline Alabama & AL & 01 & 2 & /cdroms/disk26/01 & 552 & 67 \\
\hline Alaska & AK & 02 & 1 & /cdroms/disk25/02 & 109 & 25 \\
\hline Arizona & AZ & 04 & 3 & /cdroms/disk27/04 & 538 & 15 \\
\hline
\end{tabular}




\begin{tabular}{|c|c|c|c|c|c|c|}
\hline state / counties & $a b b r$ & EIPS & call & location & MB & ncy \\
\hline Arkansas & $\mathrm{AR}$ & 05 & 4 & /cdroms/disk28/05 & 524 & 75 \\
\hline California & \multirow[t]{4}{*}{$C A$} & \multirow[t]{4}{*}{06} & & total & 1,405 & 58 \\
\hline $\begin{array}{l}\text { Alameda- } \\
\text { Mono }\end{array}$ & & & 5 & /cdroms/disk29/06 & 613 & 26 \\
\hline $\begin{array}{l}\text { Monterey- } \\
\text { Siskiyou }\end{array}$ & & & 6 & /cdroms/disk30/06 & 624 & 21 \\
\hline Solano-Yuba & & & 7 & /cdroms/disk31/06 & 168 & 11 \\
\hline Colorado & $\mathrm{CO}$ & 08 & 8 & /cdroms/disk32/08 & 498 & 63 \\
\hline Connecticut & $\mathrm{CT}$ & 09 & 9 & /cdroms/disk33/09 & 129 & 8 \\
\hline Delaware & $\mathrm{DE}$ & 10 & 9 & /cdroms/disk33/10 & 39 & 3 \\
\hline $\begin{array}{l}\text { District of } \\
\text { Columbia }\end{array}$ & $\mathrm{DC}$ & 11 & 10 & /cdroms/diak34/11 & 7 & 1 \\
\hline Florida & \multirow[t]{3}{*}{ FI } & \multirow[t]{3}{*}{12} & & total & 836 & 67 \\
\hline $\begin{array}{l}\text { Alachua- } \\
\text { Pasco }\end{array}$ & & & 11 & /cdroms/disk35/12 & 633 & 51 \\
\hline $\begin{array}{l}\text { Pinellas- } \\
\text { Washington }\end{array}$ & & & 12 & /cdroms/disk36/12 & 203 & 16 \\
\hline Georgia & GA & 13 & 13 & /cdroms/disk37/13 & 645 & 159 \\
\hline Hawaii & $\mathrm{HI}$ & 15 & 1 & /cdroms/disk25/15 & 68 & 5 \\
\hline Idaho & ID & 16 & 12. & /cdroms/disk36/16 & 377 & 44 \\
\hline Illinois & \multirow[t]{3}{*}{ II } & \multirow[t]{3}{*}{17} & & total & 732 & 102 \\
\hline $\begin{array}{l}\text { Adams - } \\
\text { Rock Island }\end{array}$ & & & 14 & /cdroms/disk38/17 & 579 & 81 \\
\hline $\begin{array}{l}\text { St. Clair- } \\
\text { woodford }\end{array}$ & & & 15 & /cdroms/disk39/17 & 153 & 21 \\
\hline Indiana & IN & 18 & 15 & /cdroms/disk39/18 & 449 & 92 \\
\hline Iowa & IA & 19 & 16 & /cdroms/disk40/19 & 429 & 99 \\
\hline Kansas & ks & 20 & 17 & /cdroms/disk $41 / 20$ & 587 & 1.05 \\
\hline Kentucky & KY & 21 & 18 & /cdroms/disk $42 / 21$ & 456 & 120 \\
\hline Louisisana & LA & 22 & 19 & /cdroms/disk $43 / 22$ & 484 & 64 \\
\hline Maine & ME & 23 & 20 & /cdroms/disk $44 / 23$ & 187 & 16 \\
\hline Maryland & $\mathrm{MD}$ & 24 & 21 & /cdroms/disk45/24 & 211 & 24 \\
\hline Massachusetts & MA & 25 & 20 & /cdroms/disk44/25 & 248 & 14 \\
\hline Michigan & MI & 26 & 22 & /cdroms/disk $46 / 26$ & 600 & 83 \\
\hline Minnesota & MN & 27 & 23 & /cdroms/disk47/27 & 556 & 87 \\
\hline
\end{tabular}




\begin{tabular}{|c|c|c|c|c|c|c|}
\hline \multicolumn{7}{|c|}{ Table IV. 1990 TIGER files: postcensus release } \\
\hline state / counties & abbr & FIPS & call & location & MB & ncy \\
\hline Mississippi & MS & 28 & 24 & /cdroms/disk $48 / 28$ & 465 & 82 \\
\hline Missouri & \multirow[t]{3}{*}{ мо } & \multirow[t]{3}{*}{29} & & total & 683 & 115 \\
\hline Adair-Texas & & & 25 & /cdroms/disk49/29 & 639 & 107 \\
\hline Vernon-Wright & & & 17 & /cdroms/disk41/29 & 44 & 8 \\
\hline Montana & MT & 30 & 26 & /cdroms/disk50/30 & 593 & 57 \\
\hline Nebraska & NE & 31 & 27 & /cdroms/disk51/31 & 400 & 93 \\
\hline Nevada & NV & 32 & 7 & /cdromsidisk31/32 & 359 & 17 \\
\hline New Hampshire & NH & 33 & 20 & /cdroms/disk44/33 & 96 & 10 \\
\hline New Jersey & $\mathrm{NJ}$ & 34 & 9 & /cdroms/disk33/34 & 263 & 21 \\
\hline New Mexico & NM & 35 & 28 & /cdroms/disk52/35 & 531 & 33 \\
\hline New York & NY & 36 & 29 & /cdroms/disk53/36 & 644 & 62 \\
\hline North Carolina & \multirow[t]{3}{*}{ NC } & \multirow[t]{3}{*}{37} & & total & 706 & 100 \\
\hline $\begin{array}{l}\text { Alamance- } \\
\text { Stokes }\end{array}$ & & & 30 & /cdroms/disk54/37 & 605 & 85 \\
\hline $\begin{array}{l}\text { Surry- } \\
\text { Yancey }\end{array}$ & & & 31 & /cdroms/disk55/37 & 101 & 15 \\
\hline North Dakota & ND & 38 & 32 & /criroms/disk56/38 & 325 & 53 \\
\hline Ohio & $\mathrm{OH}$ & 39 & 33 & /cdroms/disk57/39 & 633 & 88 \\
\hline Oklahoma & OK & 40 & 34 & /cdroms/disk58/40 & 550 & 77 \\
\hline Oregon & OR & 41 & 35 & /cdroms/disk59/41 & 590 & 36 \\
\hline Pennsylvania & \multirow[t]{3}{*}{ PA } & \multirow[t]{3}{*}{42} & & total & 778 & 67 \\
\hline $\begin{array}{l}\text { Adams- } \\
\text { Schuylkill }\end{array}$ & & & 36 & /cdroms/disk60/42 & 643 & 54 \\
\hline Snyder-York & & & 9 & /cdroms/disk33/42 & 135 & 13 \\
\hline Rhode Island & RI & 44 & 9 & /cdroms/disk33/44 & 43 & 5 \\
\hline South Carolina & sc & 45 & 31 & /cdroms/disk55/45 & 407 & 46 \\
\hline South Dakota & SD & 46 & 37 & /cdroms/disk61/46 & 369 & 66 \\
\hline Tennessee & TN & 47 & 38 & /cdroms/disk62/47 & 548 & 95 \\
\hline Texas & \multirow[t]{3}{*}{$T X$} & \multirow[t]{3}{*}{48} & & total & 1,929 & 254 \\
\hline $\begin{array}{l}\text { Anderson- } \\
\text { Fayette }\end{array}$ & & & 39 & /cdroms/disk63/48 & 614 & 75 \\
\hline $\begin{array}{l}\text { Fisher- } \\
\text { McCulloch }\end{array}$ & & & 40 & /cdroms/disk64/48 & 582 & 79 \\
\hline
\end{tabular}




\begin{tabular}{|c|c|c|c|c|c|c|}
\hline & & GTPC & נוקם & & & \\
\hline $\begin{array}{l}\text { McLennan- } \\
\text { Wheeler }\end{array}$ & & & 41 & /cdroms/disk65/48 & 642 & 88 \\
\hline $\begin{array}{l}\text { Wichita- } \\
\text { Zavala }\end{array}$ & & & 34 & /cdroms/disk58/48 & 91 & 12 \\
\hline Utah & UT & 49 & 32 & /cdroms/disk56/49 & 301 & 29 \\
\hline Vermont & VT & 50 & 20 & /cdroms/disk $44 / 50$ & 69 & 14 \\
\hline Virginia & VA & 51 & 10 & /cdroms/disk34/51 & 539 & 136 \\
\hline Washington & WA & 53 & 42 & /cdroms/disk66/53 & 549 & 39 \\
\hline West Virginia & wV & 54 & 21 & /cdroms/disk45/54 & 297 & 55 \\
\hline Wisconsin & WI & 55 & 43 & /cdroms/disk67/55 & 529 & 72 \\
\hline Wyoming & wY & 56 & 44 & /cdroms/disk68/56 & 403 & 23 \\
\hline outlying areas & & & & total & 235 & 111 \\
\hline American Samoa & AS & 60 & 1 & /cdroms/diskž, 60 & 4 & 5 \\
\hline $\begin{array}{l}\text { Federated States } \\
\text { of Micronesia }\end{array}$ & & 64 & 1 & /cdroms/disks25/64 & 7 & 4 \\
\hline Guam & GU & 66 & 1 & /cdroms/disk25/66 & 9 & 1 \\
\hline Marshall Islands & & 68 & 1 & /cdroms/disk25/68 & 5 & 33 \\
\hline $\begin{array}{l}\text { Northern Mariana } \\
\text { Islands }\end{array}$ & CM & 69 & 1 & /cdroms/disk25/69 & 4 & 4 \\
\hline Palau & & 70 & 1 & /cdroms/disk25/70 & 5 & 16 \\
\hline Puerto Rico & PR & 72 & 1 & /cdroms/disk25/72 & 193 & 78 \\
\hline $\begin{array}{l}\text { U.S Minor } \\
\text { outiying Islands } \\
\text { (Midway Islands) }\end{array}$ & & 74 & 1 & /cdroms/disk25/74 & 0.02 & 1 \\
\hline $\begin{array}{l}\text { Virgin Islands } \\
\text { of the United } \\
\text { States }\end{array}$ & VI & 78 & 1 & /cdroms/disk25/78 & 8 & 3 \\
\hline
\end{tabular}




\section{CENSUS: SUMMARY TAPE FILE 1A (STF1A)}

The locations of 1990 Census Summary Tape File 1A files, oy state, are as follows. (FIPS = Federal Information Processing System state code; location = path name in cedrcd.lbl.gov ; $\mathrm{MB}=$ size in megabytes; ncy = number of counties.)

\begin{tabular}{|l|l|l|l|r|r|}
\hline \multicolumn{5}{|c|}{ Table V. 1990 Census: Summary Tape File 1A (STF1A) } \\
\hline \hline state & abbr & FIPS & location & MB & ncy \\
\hline \hline California & CA & 06 & /cdroms/disk22/*ca.* & 656 & 58 \\
\hline
\end{tabular}

\section{CENSUS: SUMMARY TAPE FILE 3A (STF3A)}

The locations of 1990 Census Summary Tape File 3A files, by state and county, are as follows. (FIPS = Federal Information Processing System state code; location = path name in cedrcd. Ibl.gov ; $\mathrm{MB}=$ size in megabytes; ncy = number of counties.)

The present STF3A data are from a prototype CD-ROM diskette produced in May 1992. The diskette contains known errors and will be replaced later.

\begin{tabular}{||c|l|l|l|r|r|}
\hline \multicolumn{6}{|c|}{ Table VI. 1990 Census: Summary Tape File 3A } \\
\hline \hline state / counties & abbr & FIPS & location & MB & ncy \\
\hline \hline $\begin{array}{c}\text { California } \\
\text { Los Angeles }\end{array}$ & CA & 06 & & & \\
\hline
\end{tabular}




\section{NFS SERVERS}

Currently, data on CD-ROM are exported from the following NFS servers:

Lawrence Berkeley Laboratory:
parep2.lbl.gov
/home/parep2/data1
(documentation)
parep2.lbl.gov
/disk1 - /disk24
(CD-ROM data)
parep.lbl.gov
/disk25 - /disk48
(CD-ROM data)
misty.lbl.gov
/disk49 - /disk72
(CD-ROM data)
UC Data and Technical Assistance (UC DATA): csm29.berkeley.edu /disk73 - /disk84
(CD-ROM data)

Presently, /disk20, /disk21 and /disk70 - /disk84 are not used.

\section{NFS CLIENTS}

Currently, data on CD-ROM are exported to the following NFS clients. File locations as seen by the user are determined by the NFS client system administrator.

( $M=$ mounted by NFS client)

$(\mathrm{E}=$ exported, not yet mounted)

$(\mathrm{R}=$ requested, not yet exported)

Lawrenct: Berkeley Laboratory:

cedrcd.lbl.gov
parep2.lbl.gov
parep.lbl.gov
misty.lbl.gov
daisy.lbl.gov
cedr.lbl.gov
csr6.lbl.gov
ux5196.lbl.gov
csr8.lbl.gov

UC Data and Technical Assistance (UC DATA):

$\begin{array}{lll}\text { sdp2.berkeley.edu } & \text { (Sun-3) } & \text { M } \\ \text { csm29.berkeley.erlu } & \text { (SPARC) } & \text { M } \\ \text { sdp1.berkeley.edu } & \text { (PC) } & \text { E } \\ \text { CSM26.Berkeley.EDU } & \text { ('BM RISC 6000) } & \text { E }\end{array}$

UC Berkeley Regional Planning and Center for Environmental Design: redwood.ced.berkeley.edu

(SPARC)

M

sibley.ced.berkeley.edu

(SPARC)

M

iurd-j.ced.berkeley.edu

(PC)

E

dean1.ced.berkeley.edu

sonoma.ced.berkeley.edu

(PC)

E

(SPARC)

$\mathbf{R}$

UC Berkeley School of Public Health: psi.berkeley.edu

(SPARC)

E 


\section{SYSTEM ADMINISTRATORS}

The following system administrators should be contacted for technical information about specific NFS servers or NFS clients:

Lawrence Berkeley Laboratory:

Bob Rendler

(510) 486-5629

rerendler@lbl.gov

UC Data and Technical Assistance (UC DATA):

Norm Gelbart (510) 643-9132 nbg@alfa.berkeley.edu

UC Berkeley Regional Planning and Center for Environmental Design:

James Ganong

(510)643-5048 jeg@ced.berkeley.edu

Bob Higbie

(510) 643-5047

bobhig@ced.berkeley.edu

UC Berkeley School of Public Health:

Bob Sholtz

(510) $642-5030$

sholtz@psi.berkeley.edu

\section{ACKNOWLEDGMENTS}

The author wishes to acknowledge assistance and support provided by Harvard Holmes, Fred Gey, Carl Quong, Bob Rendler, Norm Gelbart, Gary Peete, and Mari Miller.

This work was supported by the Director, Office of Epidemiology and Health Surveillance, Office of Health, Office of Environment, Safety and Health, U.S. Department of Energy under Contract No. DE-AC03-76SF00098. 


\section{FURTHER INFORMATION}

For further information, contact:

Deane Merrill

Information and Comfuting Sciences Division

Building 50B, Room 3229

Lâwrence Berkeley Laboratory

Berkeley CA 94720

tel: (510) 486-5063

fax: (510) 486-6363

internet: dwmerrillelbl.gov

bitnet: merrillelil (merrillelbl.bitnet)

compuserve: 71001,62 (71001.62@compuserve.com)

\section{OBTAINING THIS DOCUMENT}

This document is available via anonymous FTP from

Internet node:

directory:

DOS WordPerfect 5.1:

PostScript:

ASCII text: cedrcd.1bl.gov

/data1/merrill/docs/cedr

cdrom1.wp5

cdrom $1 . p s$

cdroml.asc

For example, to obtain cdrom $1 . p s$ for printing on your computer, enter:

ftp cedrcd.lbl.gov

anonymous

(user name)

dwmerrillelbl.gov (instead of password, your own $\mathrm{ID}$ in this format)

cd /datal/merrill/docs/cedr

dir

(to see names and sizes of files)

binary

get corom 1.ps

(required for carom1.wp5)

quit

The same document in Macintosh Microsoft Word 5.0 format is in LBL AppleTalk zone icsd, Public Folder DWMerrill, file Census Data on CD-ROM .

Future revisions will be stored in the same locations and will be announced in the LBL Computing Newsletter.

This version is csr6.1bl.gov: c: \docs \cedr\cdrom $1.217 / 2 / 92$ 

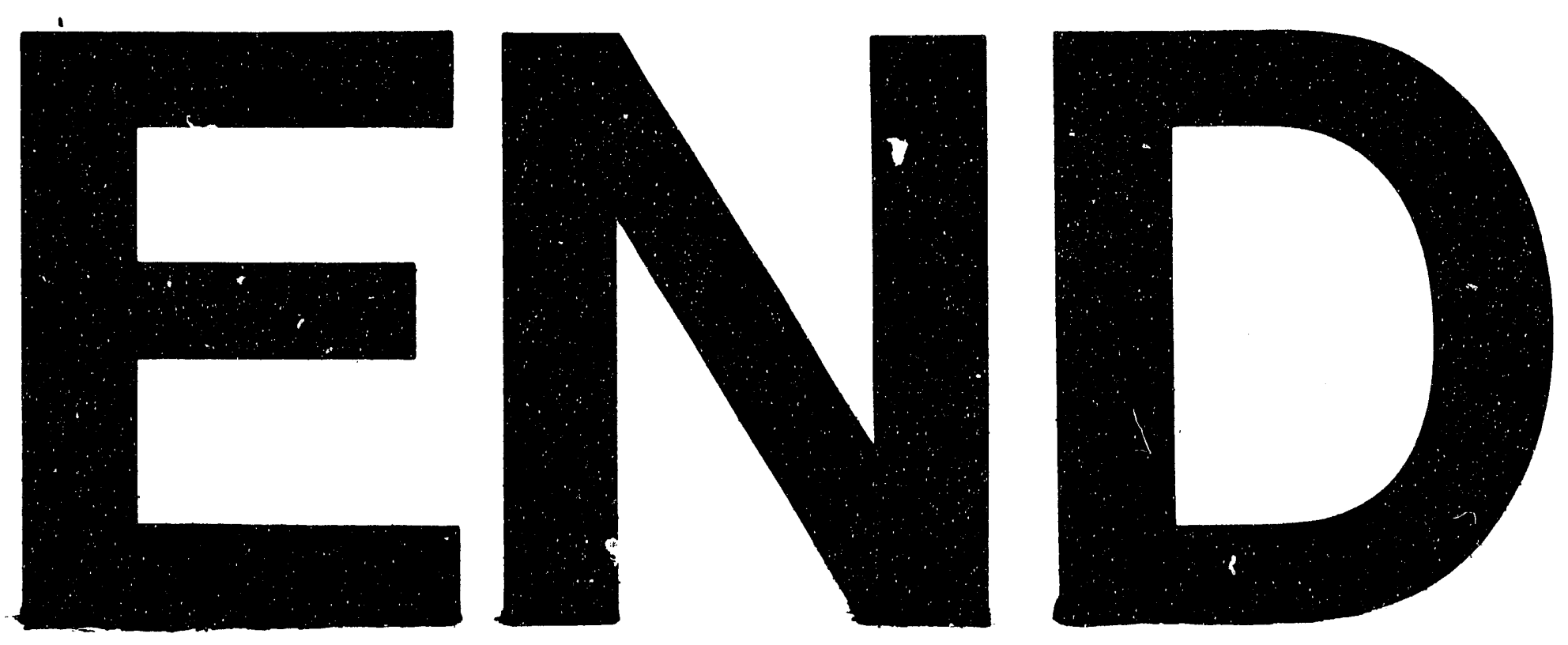

2

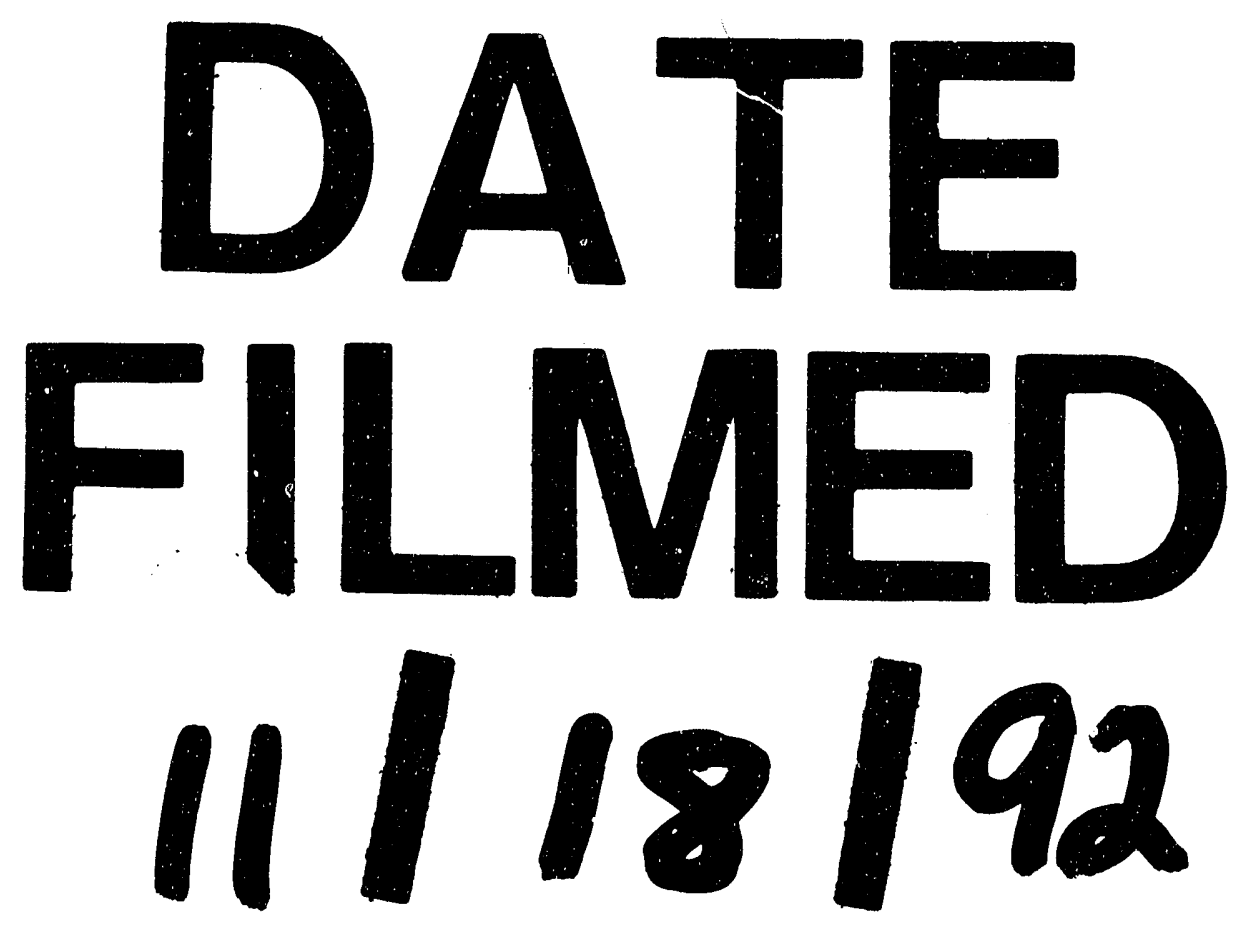


